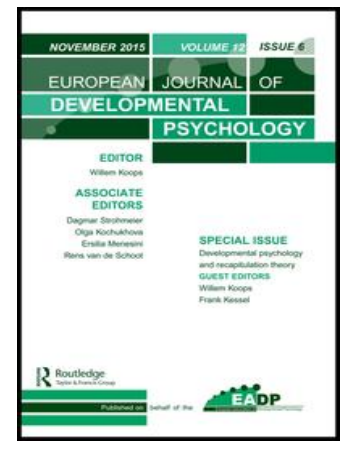

European Journal of Developmental Psychology

\title{
Parent-adolescent relationships: an adjusted person-centred approach
}

Hana Hadiwijaya, Theo A. Klimstra, Jeroen K. Vermunt, Susan J. T. Branje \& Wim H. J. Meeus

To cite this article: Hana Hadiwijaya, Theo A. Klimstra, Jeroen K. Vermunt, Susan J. T. Branje \& Wim H. J. Meeus (2015) Parent-adolescent relationships: an adjusted personcentred approach, European Journal of Developmental Psychology, 12:6, 728-739, DOI: 10.1080/17405629.2015.1110519

To link to this article: https://doi.org/10.1080/17405629.2015.1110519

册 Published online: 26 Nov 2015.

Submit your article to this journal $₫$

Щ Article views: 361

View Crossmark data ¿

Citing articles: 1 View citing articles $\square$ 


\title{
DEVELOPMETRICS
}

\section{Parent-adolescent relationships: an adjusted person- centred approach}

\author{
Hana Hadiwijaya ${ }^{\text {a }}$,Theo A. Klimstra ${ }^{\text {a }}$, Jeroen K. Vermunt ${ }^{\text {b }}$ Susan J. T. Branje \\ and Wim H. J. Meeus ${ }^{\mathrm{a}, \mathrm{c}}$ \\ aDepartment of Developmental Psychology, Tilburg University, Tilburg, The Netherlands; \\ ${ }^{b}$ Department of Methodology and Statistics, Tilburg University, Tilburg, The Netherlands; \\ 'Adolescent Development, Utrecht University, Utrecht, The Netherlands
}

\begin{abstract}
Person-centred approaches classify individuals with similar attributes in the same group and describe differences between these groups of individuals. However, these approaches are scarcely used, partly due to their low predictive power. This study aimed to overcome previous limitations using an adjusted personcentred procedure to identify a reliable and valid parent-adolescent typology and demonstrate the value of an adjusted approach. Adolescents ( $N=2281,49 \%$ males, $M_{\text {age }}=14.35, \mathrm{SD}_{\text {age }}=2.33$ ) completed self-reports regarding relationship quality, psychopathology and personality. Harmonious, average, turbulent and detached relationship types were identified and replicated. These types showed external validity, as they displayed distinctive patterns in psychopathology and personality. The adjusted procedure clearly increased predictive power, as it explained more variance in outcomes when compared to the standard procedure. This study contributes to adolescent research by identifying a reliable and valid parent-adolescent relationship typology and demonstrating the value of an adjusted person-centred approach.
\end{abstract}

\section{KEYWORDS Parent-adolescent relationships; person-centred approach}

Similar to personality profiles, relationship profiles can be viewed as dynamic organizations in which relational components function as configured rather than isolated systems (Allport, 1937). Person-centred approaches (e.g., cluster analysis, latent class analysis) produce such profiles by grouping individuals into classes using configurations of components in which each class includes individuals who are similar to each other and different from those in other classes (Asendorpf, 2006). For instance, configurations of responsiveness and demandingness revealed a parenting typology including authoritative (responsive and 
Table 1. An example of three individuals (A, B, C) having non-zero classification probabilities for each of the three classes.

\begin{tabular}{lccc}
\hline & \multicolumn{3}{c}{ Classification probabilities } \\
\hline & Class 1 & Class 2 & Class 3 \\
\hline Individual A & 0.80 & 0.10 & 0.10 \\
Individual B & 0.10 & 0.75 & 0.15 \\
Individual C & 0.15 & 0.15 & 0.70 \\
\hline
\end{tabular}

Notes: Rows display the classification probabilities. In this example, individual A has $80 \%$ chance to belong to Class 1, 10\% chance to belong to Class 2 and $10 \%$ chance for Class 3. In general, individuals are assigned to the class for which the classification probability is the largest (individual A would be assigned to Class 1, B to Class 2 and C to Class 3). However, as they do not have a $100 \%$ probability to belong to the assigned class, their class assignments are imperfect and include classification errors.

demanding), authoritarian (non-responsive and demanding), mid-range (moderate responsive and demanding), indulgent (responsive and undemanding) and negligent (non-responsive and undemanding) parenting profiles (e.g., Baumrind, 1991; Slicker, 1998).

However, the person-centred approaches that are typically used (e.g., cluster analysis, latent class analysis) are plagued by low predictive power due to individual classification errors. That is, individuals assigned to a class may also express some characteristics of other classes (Asendorpf, 2006), as illustrated in Table 1. Because classes may include inaccurately assigned individuals, this introduces classification errors. No adjustment for these errors produces low predictive power and limits the identification of linkages of profiles with, for instance, behavioural problems. Therefore, adjustment for the classification errors inherent to person-centred approaches is needed. A newly developed three-step procedure addresses this limitation by adjusting for classification inaccuracy, thereby providing greater predictive power (e.g., Bakk, Tekle, \& Vermunt, 2013; Vermunt, 2010).

Partly because of the limited predictive power of unadjusted person-centred approaches, most relationship research has applied variable-centred approaches. Such variable-centred approaches (e.g., correlations, regression analysis) focus on associations between variables (e.g., linking interindividual differences in parent-adolescent quality to interindividual differences in well-being) and not on configurations of characteristics. Thereby, these approaches ignore the notion that relationships are structured as dynamic organizations (Allport, 1937). A more comprehensive understanding of parent-adolescent relationships will likely be obtained by applying an adjusted person-centred approach to define a typology in which configurations of relationship components are preserved.

To build a typology of parent-adolescent relationships, three key components defining this relationship are of importance: power, support and conflict (Furman \& Buhrmester, 1985). Power represents dominance and equality, support refers to nurturance and prosocial behaviour and conflict includes negative interactions and antagonism. These components are also represented in 
Steinberg and Silk's (2002) parent-adolescent relationship domains and are often used in conceptualizations of parent-adolescent relationships (e.g., De Goede, Branje, \& Meeus, 2009).

Until now, no study has applied a person-centred approach to identify a typology of parent-adolescent relationships using all these key components. Relatedly, in many developmental studies, classification errors remain unaccounted for if person-centred approaches are applied. In addition, no study has yet demonstrated the incremental value of an adjusted approach in which classification errors are accounted for, over the standard approach in which these errors remain unaccounted for. Therefore, the aim of this study was twofold: (1) applying an adjusted person-centred procedure to identify a reliable and valid global parent-adolescent typology, and (2) investigating the merits of an adjusted approach compared to the standard approach.

As no research has identified a typology using the three key relationship components, we based our hypothesis on the extended parenting typology of Baumrind (1991), McKinney and Renk (2008) and Slicker (1998) in which only responsiveness (similar to support) and demandingness (similar to power) were configured. We expected to find relationship types representing authoritative (high on support and power), authoritarian (low on support, high on power), mid-range (moderate support and power), indulgent (high on support, low on power) and negligent (low on support and power) profiles. Furthermore, we expected that relationship types derived with the adjusted approach would provide greater predictive power than relationship types derived with the standard approach (Vermunt, 2010).

\section{Method}

\section{Participants and procedure}

Data for the current study were collected as part of a Dutch longitudinal project Conflict and Management of Relationships (CONAMORE) approved by the local institutional review board. Initially, 2391 adolescents participated. Adolescents with missing values on relationship quality variables were excluded from the analyses as these variables defined our typology. Adolescents with missing values on other variables were included in the analyses. Little's (1988) Missing Completely at Random test indicated that the data were likely missing at random $(\chi 2 / d f=1.90 ;$ Bollen, 1989), suggesting that adolescents with missing data were similar to those with complete data. This justifies our approach to handling missing data.

The final sample included 2281 adolescents (49\% males, $M_{\text {age }}=14.35$, $\mathrm{SD}_{\text {age }}=2.33$ ), including two age groups: early-to-middle adolescents ( $n=1293$; $51 \%$ males, $M_{\text {age }}=12.45, \mathrm{SD}_{\text {age }}=0.61$, range $10-15$ years) and middle-to-late adolescents $\left(n=988 ; 43 \%\right.$ males, $M_{\text {age }}=16.87, \mathrm{SD}_{\text {age }}=0.98$, range $16-24$ years). 
Adolescents were recruited from various high schools in the province of Utrecht, the Netherlands, and they were all in junior high and high schools. Most participants were Dutch (81\%), whereas others belonged to the largest ethnic minorities in the Netherlands (e.g., Surinamese, Antillean, Moroccan).

\section{Measures}

Comprehensive information regarding all measures can be found in Table 1 of the supplementary material. This table provides example items and psychometric properties for each measure.

\section{Relationship quality}

The Network of Relationships Inventory (Furman \& Buhrmester, 1985) was used to measure adolescents' perceptions of adolescent-mother and adolescentfather relationship quality based on parental power ( 6 items), support ( 12 items) and conflict ( 6 items) on a 5-point Likert scale. Scores for adolescent-mother and adolescent-father relationship quality on each component were collapsed, as our study aimed to identify a global relationship typology. Principal component analysis showed that the underlying factors represented the three NRI components rather than different adolescent-mother or adolescent-father relationship factors.

\section{Psychopathology}

The Children's Depression Inventory (Kovacs, 1985) was used to measure adolescents' depressive symptoms on a 3-point Likert scale (27 items). The Screen for Child Anxiety Related Emotional Disorders (Birmaher et al., 1997) was used to measure anxiety on a 3-point Likert scale (38 items). The Direct and Indirect Aggression Scale (Björkqvist, Lagerspetz, \& Kaukiainen, 1992) was used to measure direct aggression (5 items) and indirect aggression (12 items) on a 4-point Likert scale.

\section{Personality}

The 30-item Quick Big Five (Vermulst \& Gerris, 2005) was used to measure adolescents' personality traits of Openness, Conscientiousness, Extraversion, Agreeableness and Emotional Stability. Each trait was assessed by six items on a 7-point Likert scale.

\section{Analytic strategy}

Analyses were performed in Latent GOLD 5.0 (Vermunt \& Magidson, 2013). Specifically, a three-step adjusted person-centred procedure was performed to identify a parent-adolescent relationship typology and demonstrate the value of this approach compared to the standard approach (i.e., a latent class 
analysis in which no classification errors were taken into account) (Asparouhov \& Muthén, 2014; Bakk et al., 2013).

First, a latent class analysis (LCA) was conducted to identify a parent-adolescent relationship typology. LCA is a person-centred analytic method that groups individuals into classes based on their pattern of scores across variables (i.e., power, support and conflict). Similar to cluster analysis, LCA generates measurement and structural parameters (Nylund, Asparouhov, \& Muthén, 2007), but it also offers fit statistics and significance tests to identify the number of classes. Class membership assignment is determined based on class probabilities. Three criteria were used to determine the number of latent classes: the Bayesian information criterion (BIC; Schwarz, 1978) should be the lowest, the solution with $k+1$ class should lead to an improvement in model fit as indicated by a significant bootstrap/likelihood ratio (BLRT; Nylund et al., 2007), and the most parsimonious solution should be selected if an additional class in a $k$ class model represented a variation of a solution with $k-1$ class.

In the second step, we computed the probability of belonging to each of the classes using adolescents' scores on power, support and conflict. These classification probabilities were subsequently used to assign each adolescent to the class for which the classification probability was largest. Note that classifications would be perfect if the largest classification probability equals 1 for each adolescent. As this is clearly not the case, classification errors are introduced when assigning individuals to classes. However, classification error probabilities can be computed and used in the adjustment procedure applied in the third step. For instance, when computing the mean of the first class, the procedure takes into consideration that adolescents belonging to the first class also have a certain probability to be assigned to the second, third or fourth class. The procedure adjusts for such classification errors by reweighting adolescents' assigned class memberships by the inverse of the misclassification probabilities.

In the third step, the adjusted classifications were used in an ANOVA to estimate differences between relationship types on psychopathology and personality and examine the predictive power of the types on these variables, controlled for gender and age. Note that other analyses using the adjusted classifications can also be performed in the third step (e.g., multilevel analyses, regressions), as these classifications can be used to estimate the association between the latent variable (i.e., relationship class membership) and other variables (i.e., psychopathology and personality) (Bakk et al., 2013).

\section{Results}

Results of the first step are shown in Table 2 in which solutions up to six classes led to lower BIC and significant BLRT values, suggesting that each additional class contributed to model fit improvement. However, the five-class solution appeared to be the most parsimonious, as the sixth class appeared to be a 
Table 2. Latent class analysis model fit indicators up to six classes.

\begin{tabular}{lcccc}
\hline Class solutions & Log likelihood & BIC $^{\text {a }}$ & BLRT $p$-value $^{\mathrm{b}}$ & Entropy \\
\hline 1 & -6306.80 & 12660.10 & 0.00 & 1.00 \\
2 & -5782.51 & 11665.54 & 0.00 & 0.54 \\
3 & -5282.65 & 10719.94 & 0.00 & 0.68 \\
4 & -5100.17 & 10409.11 & 0.00 & 0.66 \\
5 & -4970.05 & 10202.99 & 0.00 & 0.69 \\
6 & -4866.10 & 10048.97 & 0.00 & 0.69 \\
\hline
\end{tabular}

aBayesian information criteria.

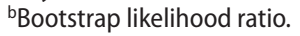
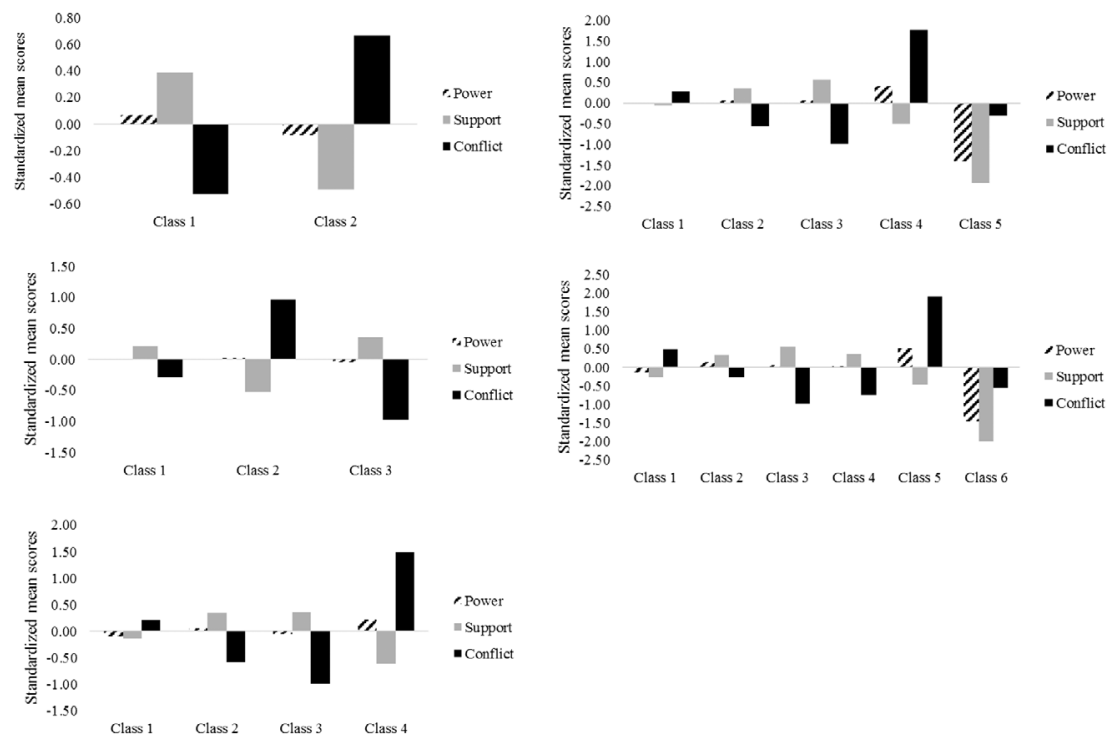

Figure 1. Parent-adolescent relationship profiles for latent class solutions up to six classes based on perceived parental power, support and parent-adolescent conflict $(N=2281)$.

slight variation of one of the classes in the five-class solution (see Figure 1). The four-class solution was rejected as it showed a worse model fit than the fiveclass solution and missed a unique class that the five-class solution provided. Therefore, the five-class solution was chosen as the final model. Additionally, the second and third class of this five-class solution were merged into one class as they were very similar to each other (Hennig, 2010). The final typology was thus a five-class solution integrated into four classes (Figure 2). The entropy value of this four-class model was acceptable (.64). To ensure that the current solution was reliable, the total sample was randomly split $(I=1160 ; I I=1121)$ and identical classes were obtained as shown in Figures 1 and 2 of the supplementary material. The classes we found were a harmonious class (48\%; average levels of power, high levels of support and low levels of conflict), an average class (38\%; levels of power, support and conflict around the sample mean), a turbulent class 


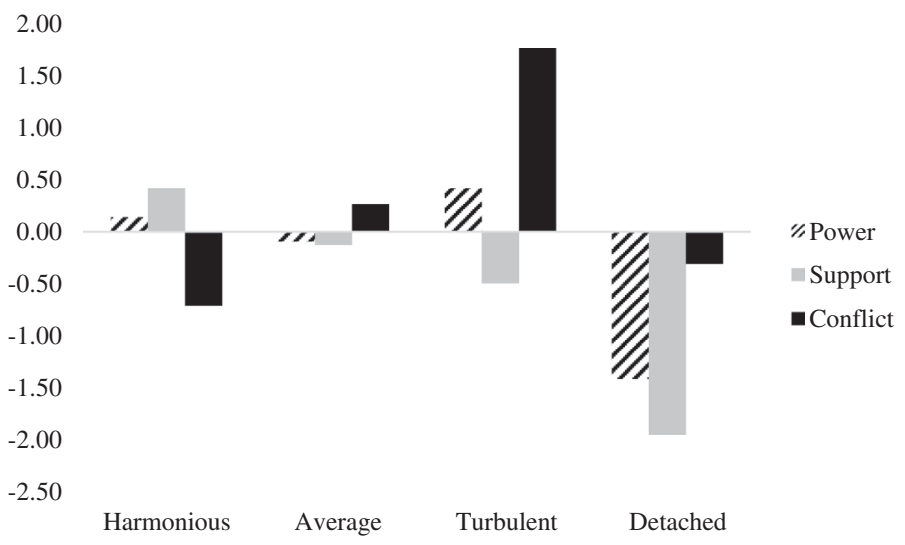

Figure 2. Integrated four-class solution profiles of parent-adolescent relationships based on perceived parental power, support and parent-adolescent conflict $(N=2281)$. Parental power and support scores of the average class and parental power scores of the harmonious class were slightly adjusted as values of these classes were on a similar level as the $x$-axis and thus barely visible.

Table 3. Classification Assignments for the Integrated Four-class Solution.

\begin{tabular}{lcccc}
\hline & Harmonious & Average & Turbulent & Detached \\
\hline Harmonious & 824.65 & 141.78 & 9.15 & 8.81 \\
Average & 141.78 & 646.62 & 80.18 & 21.49 \\
Turbulent & 9.61 & 80.18 & 189.57 & 4.93 \\
Detached & 12.13 & 21.49 & 4.93 & 79.93 \\
Total & 988.16 & 890.06 & 284.29 & 118.48 \\
\hline
\end{tabular}

Notes: This table shows fraction numbers of assigned individuals as classifications were based on proportional assignments in which individual probabilities of belonging to each of the four classes were accounted for.

(9\%; high levels of power and conflict, low levels of support) and a detached class (5\%; low levels of all relationship quality variables).

Table 3 shows the class assignments derived from the second step. When computing the probability of belonging to each of the classes using adolescents' scores on relationship quality, the probabilities of the second and third class from the five-cluster solution were summed in the current four-class typology.

Results of the third step can be found in Table 4, which also displays the sample size and mean scores of relationship quality for each relationship type. The harmonious and average class displayed similar levels of power, but other than that all types displayed significantly different patterns of relationship quality. Table 4 also shows that adolescents with a harmonious relationship displayed the least psychopathology and the best-adjusted personality profile (i.e., highest scores on personality traits). Additionally, adolescents in an average relationship showed a profile in between the other classes. Adolescents with a turbulent relationship displayed more psychopathology and a less-adjusted personality 


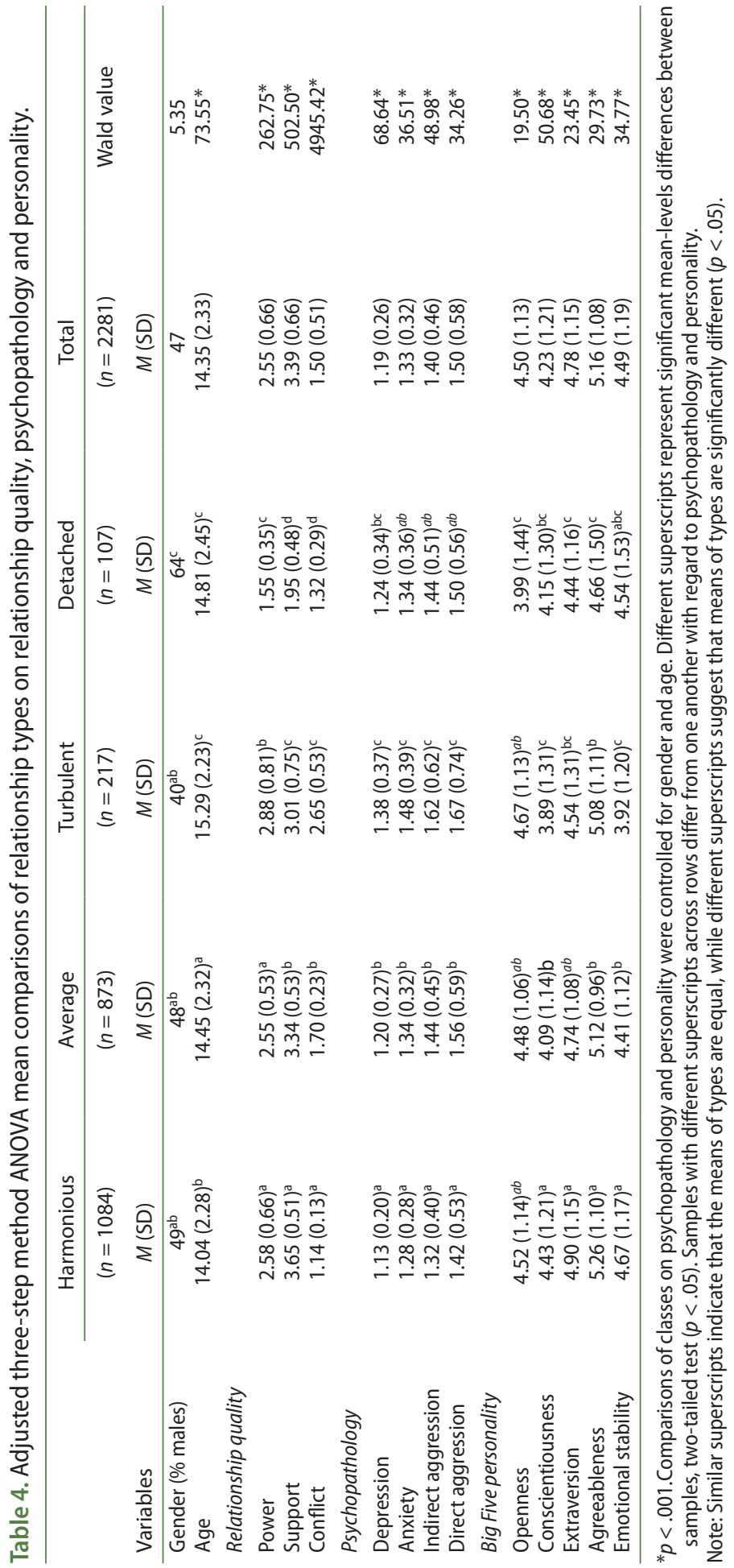


Table 5. Explained variance of parent-adolescent relationship typology on psychopathology and personality using the standard and adjusted approach.

\begin{tabular}{lcc}
\hline & Standard approach $R^{2}$ & Adjusted approach $R^{2}$ \\
\hline Psychopathology & & \\
Depression & 0.08 & 0.13 \\
Anxiety & 0.04 & 0.05 \\
Indirect aggression & 0.04 & 0.07 \\
Direct aggression & 0.02 & 0.04 \\
Big Five personality & & \\
Openness & 0.01 & 0.02 \\
Conscientiousness & 0.03 & 0.05 \\
Extraversion & 0.01 & 0.03 \\
Agreeableness & 0.02 & 0.03 \\
Emotional stability & 0.03 & 0.05 \\
\hline
\end{tabular}

Notes: The standard approach was conducted using an ANOVA in SPSS 19.0 in which no classification errors were taken into account, whereas these errors were considered for the ANOVA performed with the threestep procedure in Latent GOLD 5.0.

profile than adolescents with harmonious or average relationships. Adolescents in a detached relationship showed less psychopathology than those in a turbulent relationship, but were also less open and agreeable. Moreover, Table 5 shows that the adjusted procedure systematically explained more variance (i.e., almost twice as much) as the standard approach in which classification errors remained unaccounted for.

\section{Discussion}

Applying an adjusted person-centred approach to three key relationship components identified four replicable parent-adolescent relationship types. These types also showed external validity as they were systematically linked to psychopathology and personality. Additionally, the adjusted person-centred approach proved to enhance the predictive power of the types when compared to an unadjusted standard approach.

Our findings partly support our hypothesis based on a parenting typology (Baumrind, 1991; Slicker, 1998), as we identified types representing mid-range (average), authoritarian (turbulent) and negligent (detached) profiles. However, the harmonious profile fell in between the authoritative and indulgent parenting type as adolescents in this relationship type reported that their parents were supportive, and nor strict nor permissive. This type might represent an egalitarian relationship in which adolescents experience reciprocity and equality in their interactions with parents (Youniss \& Smollar, 1985). Overall, our typology seems to be a meaningful addition next to Baumrind's (1991) and McKinney and Renk (2008) parenting typology, as it specifically concerned a typology of relationship quality and also enclosed the conflict aspect of relationships. 
Furthermore, we found evidence for the adjusted procedure explaining more variance in psychopathology and personality than the standard procedure. This indicates that the adjusted approach enhanced the predictive power of relationship types. Our study thus overcame a major problem in previous person-centred studies: low predictive power (e.g., Asendorpf, 2006). This method therefore likely brightens the future of person-centred research.

Two notions warrant mention. First, most adolescents perceived a harmonious or average relationship with their parents, and only $14 \%$ experienced a turbulent or detached relationship. Our findings therefore support the modified storm-and-stress theory (Arnett, 1999) by showing that only a subgroup of adolescents experienced a turbulent relationship with their parents. Second, a detached relationship was not associated with increased risk for psychopathology, but was related to a less open and less agreeable personality. Traits representing a rigid personality may thus be linked to less close relationships. This finding is similar to previous research, in which an avoidant attachment style was characterized by a less compassionate personality (e.g., Noftle \& Shaver, 2006).

An important limitation of the current study is the global examination of parent-adolescent relationship types rather than examining and/or combining unique maternal and paternal relationship characteristics with adolescent. Some adolescents could, for instance, have good relationships with mothers, but worse relationships with fathers or vice versa (e.g., Youniss \& Smollar, 1985). Nevertheless, a global approach of a parent-adolescent relationship typology seemed an appropriate starting point to demonstrate the merits of an adjusted person-centred method for adolescent relationship research. Future research is needed to explore other potential typologies of adolescents' relationships. Additionally, the developmental patterns and outcomes of relationship types throughout the adolescence need to be investigated.

This study has several important implications. First and foremost, we demonstrated the value of an adjusted person-centred approach by accounting for classification errors and thereby increasing the predictive power of relationship types. Second, our typology provides a better understanding of parent-adolescent relationships by considering the configuration of all key relationship components simultaneously. These typologies are also easy to communicate to health care professionals interested in relationship characteristics putting adolescents at risk for psychopathology, as typological classifications (e.g., diagnoses) are frequently used in applied settings.

\section{Disclosure statement}

No potential conflict of interest was reported by the authors. 


\section{References}

Allport, G. W. (1937). Personality: A psychological interpretation. New York, NY: Holt.

Arnett, J. J. (1999). Adolescent storm and stress, reconsidered. American Psychologist, 54, 317-326.

Asendorpf, J. B. (2006). Typeness of personality profiles: A continuous person-centred approach to personality data. European Journal of Personality, 20, 83-106.

Asparouhov, T., \& Muthén, B. (2014). Auxiliary variables in mixture modeling: Three-step approaches using Mplus. Structural Equation Modeling: A Multidisciplinary Journal, 21, 329-341.

Bakk, Z., Tekle, F. B., \& Vermunt, J. K. (2013). Estimating the association between latent class membership and external variables using bias-adjusted three-step approaches. Sociological Methodology, 43, 273-311.

Baumrind, D. (1991). The influence of parenting style on adolescent competence and substance use. The Journal of Early Adolescence, 11, 56-95.

Birmaher, B., Khetarpal, S., Brent, D., Cully, M., Balach, L., Kaufman, J., \& Neer, S. M. (1997). The screen for child anxiety related emotional disorders (SCARED): Scale construction and psychometric characteristics. Journal of the American Academy of Child and Adolescent Psychiatry, 36, 545-553.

Björkqvist, K., Lagerspetz, K. M. J., \& Kaukiainen, A. (1992). Do girls manipulate and boys fight? Developmental trends in regard to direct and indirect aggression. Aggressive Behavior, 18, 117-127.

Bollen, K. (1989). Structural equations with latent variables. New York, NY: Wiley.

De Goede, I. A., Branje, S. T., \& Meeus, W. J. (2009). Developmental changes in adolescents' perceptions of relationships with their parents. Journal of Youth and Adolescence, 38, 75-88.

Furman, W., \& Buhrmester, D. (1985). Children's perceptions of the personal relationships in their social networks. Developmental Psychology, 21, 1016-1024.

Hennig, C. (2010). Methods for merging Gaussian mixture components. Advances in Data Analysis and Classification, 4, 3-34.

Kovacs, M. (1985). The children's depression inventory (CDI). Psychopharmacology Bulletin, 21, 995-998.

Little, R. J. A. (1988). A test of missing completely at random for multivariate data with missing values. Journal of the American Statistical Association, 83, 1198-1202.

McKinney, C., \& Renk, K. (2008). Differential parenting between mothers and fathers: Implications for late adolescents. Journal of Family Issues, 29, 806-827.

Noftle, E. E., \& Shaver, P. R. (2006). Attachment dimensions and the big five personality traits: Associations and comparative ability to predict relationship quality. Journal of Research in Personality, 40, 179-208.

Nylund, K. L., Asparouhov, T., \& Muthén, B. (2007). Deciding on the number of classes in latent class analysis and growth mixture modeling: A Monte Carlo simulation study. Structural Equation Modeling: A Multidisciplinary Journal, 14, 535-569.

Schwarz, G. (1978). Estimating the dimension of a model. The Annals of Statistics, 6, 461-464.

Slicker, E. (1998). Relationship of parenting style to behavioral adjustment in graduating high school seniors. Journal of Youth and Adolescence, 27, 345-372.

Steinberg, L., \& Silk, J. S. (2002). Parenting adolescents. In M. H. Bornstein (Ed.), Handbook of parenting: Vol. 1: Children and parenting (2nd ed., pp. 103-133). Mahwah, NJ: Lawrence Erlbaum Associates.

Vermulst, A. A., \& Gerris, J. R. M. (2005). QBF: Quick Big Five persoonlijkheidstest handleiding [Quick big five personality test manual]. Leeuwaarden: LDC Publications. 
Vermunt, J. K. (2010). Latent class modeling with covariates: Two improved three-step approaches. Political Analysis, 18, 450-469.

Vermunt, J. K., \& Magidson, J. (2013). Latent GOLD 5.0 Upgrade Manual.

Youniss, J., \& Smollar, J. (1985). Adolescent relations with mothers, fathers, and friends.

Chicago, IL: The University of Chicago Press. 